\title{
HR-STEM Imaging and EELS Characterizing of Nano-Scale Defects in Sputter Deposited Thin Films of Double-Perovskite $\mathrm{Sr}_{2} \mathrm{FeMoO}_{6}$ (SFMO) and $\mathrm{Sr}_{2} \mathrm{CrReO}_{6}$ (SCRO)
}

\author{
Robert E. A. Williams*, Adam J. Hauser**, Rebecca A. Ricciardo***, Manisha Dixit*, Jeremy M. \\ Lucy**, Patrick M. Woodward***, Fengyuan Yang**, Hamish L. Fraser* \\ *Department of Materials Science and Engineering, The Ohio State University \\ ** Department of Physics, The Ohio State University \\ ***Department of Chemistry, The Ohio State University
}

Oxides are promising candidates in the emerging field of spintronics. Some oxide systems of interest include double perovskites like $\mathrm{Sr}_{2} \mathrm{FeMoO}_{6}$ (SFMO) and $\mathrm{Sr}_{2} \mathrm{CrReO}_{6}$ (SCRO). $\mathrm{Sr}_{2} \mathrm{FeMoO}_{6}$ is the most studied half-metallic double perovskite with the potential for room-temperature magnetoelectronic applications due to its Curie temperature being above $400 \mathrm{~K}[1]$. However, $\mathrm{Sr}_{2} \mathrm{FeMoO}_{6}$ has not been incorporated into devices that reflect its high spin polarization largely due to lack of understanding of some fundamental questions about the fabrication of $\mathrm{Sr}_{2} \mathrm{FeMoO}_{6}$ films. Due to the complexity in the $A_{2} \mathrm{BB}^{\prime} \mathrm{O}_{6}$ double perovskites and their stringent requirements for growth conditions, it is rather challenging to obtain stoichiometric, phase pure, and fully epitaxial $\mathrm{Sr}_{2} \mathrm{FeMoO}_{6}$ films with high double perovskite ordering between B and B' sites; all of these properties are essential to achieve half-metallicity[2-6].

The magnetic properties of these systems have also been found to depend on the amount of disorder present as well as other defects. The major challenge during layer deposition is obtaining the necessary long-range ordering while preventing defect formation due to processing parameters. This requires structural and chemical characterization ranging from identification of defects and morphology as well as correlating microstructure-property relationships.

For this work thin films of SFMO and SCRO have been sputter deposited on (100) and (111) STO with varying deposition and sputtering parameters in an attempt to produce optimal electronic properties within the oxide layer. HAADF-STEM imaging and EELS analyses were conducted using an aberrationcorrected FEI Titan ${ }^{\mathrm{TM}}$ 80-300 STEM to provide microstructural and chemical information. The first HAADF-STEM images of SFMO and SCRO double perovskite ordering are presented as well as characterization of interfacial defects (FIG. 1-3) [7]. Initial EDX results were also collected using a FEI Osiris TEM to map the chemical nature of the defects present. Defect images are also compared with STEM multislice simulations for validation

Special care was taken in preparing (100) and (110) cross - sectional TEM samples for HR-STEM using a Helios Nanolab for bulk trenching and thinning. The foil was extracted using an Omniprobe 200 micromanipulator and mounted on a $\mathrm{Cu}$ grid in a flag orientation for subsequent low $\mathrm{kV}$ milling at $500 \mathrm{eV}$ to remove amorphous ion damage and provide optimal surface quality for high resolution analysis.

\section{References}

[1] Krockenberger, Y., et al., $\mathrm{Sr}_{2} \mathrm{CrOsO}_{6}$ : End Point of a Spin-Polarized Metal-Insulator transition by $5 d$ Band Filling. Phys. Rev. B 75, 020404(R) (2007).

[2] Pickett, W. E. \& Singh, D. J., Electronic Structure and Half-Metallic Transport in the $\mathrm{La}_{1-x} \mathrm{Ca}_{x} \mathrm{MnO}_{3}$ System. Phys. Rev. B 53, 1146-1160 (1996).

[3] Bibes, M. \& Barthélémy, A., Oxide Spintronics. IEEE Trans. Electron Devices 54, 1003-1023 (2007).

[4] Brabers, V. A. M., Progress in spinel ferrite research. Ferromagnetic Materials, Vol. 8, edited by Buschow, K. H. J. (Elsevier, Amsterdam,1995) p. 189.

[5] Kobayashi, K. L., Kimura, T., Sawada, H., Terakura, K., \& Tokura, Y., Room-Temperature Magnetoresistance in an Oxide Material with an Ordered Double-Perovskite Structure. Nature 395, 677-680 (1998). 
[6] Serrate, D., DeTeresa, J. M., \& Ibarra, M. R., Double Perovskites with Ferromagnetism above Room Temperature. J. Phys. Cond. Matter 19, 023201 (2007).

[7] Hauser, A.J., et. al. Unlocking the potential of half-metallic $\mathrm{Sr}_{2} \mathrm{FeMoO}_{6}$ films through controlled stoichiometry and double-perovskite ordering. Physical Review: B (To be published)
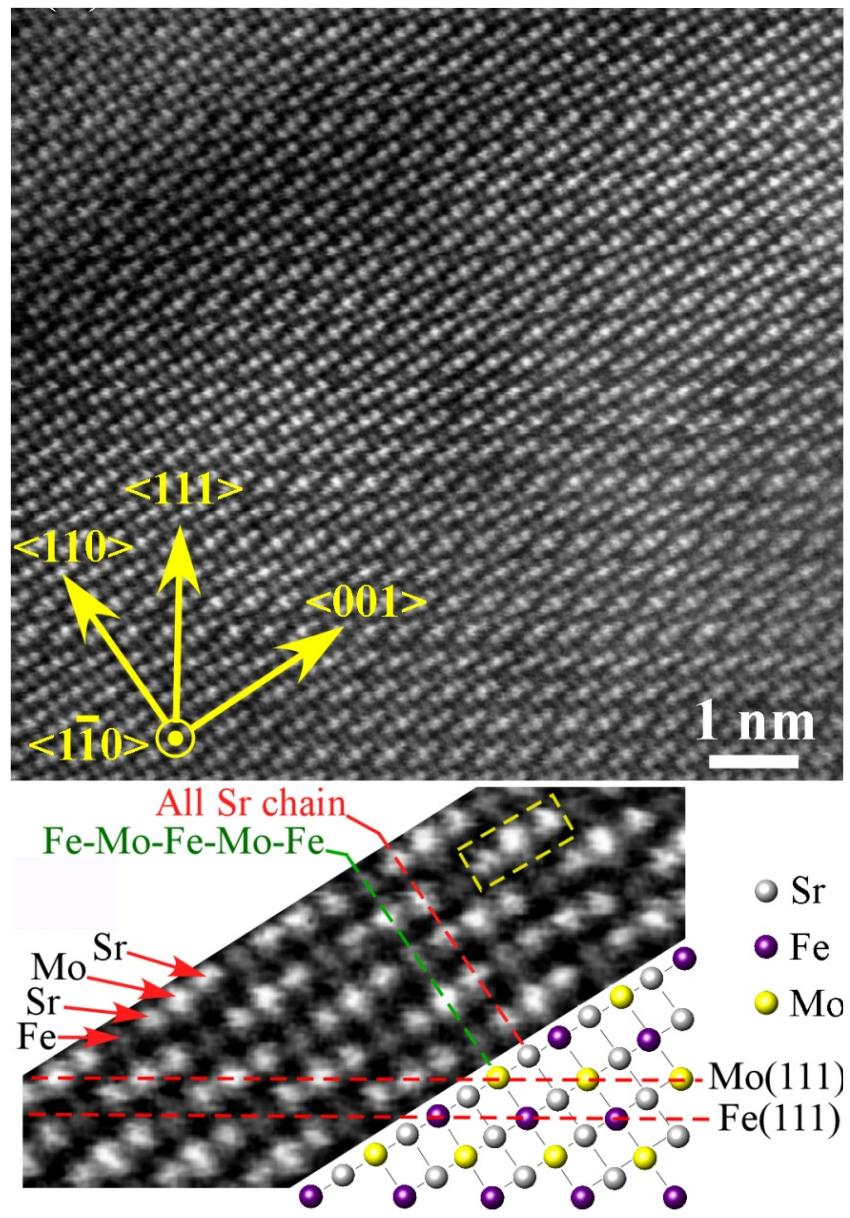

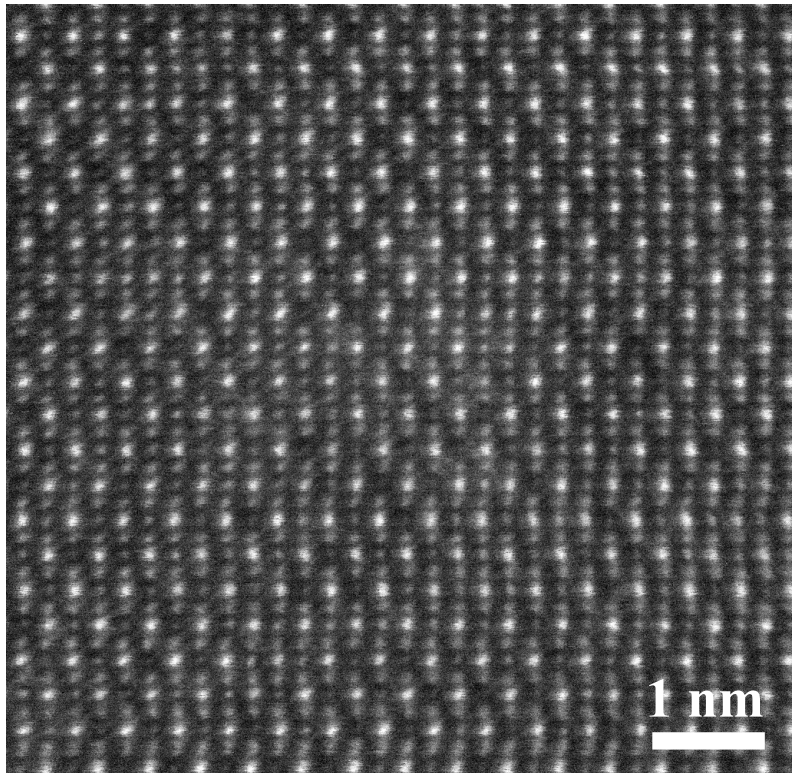

FIG. 2: HAADF-STEM image of double perovskite ordering for (110) SCRO

FIG. 1: HAADF-STEM image of double perovskite ordering for (110) SFMO
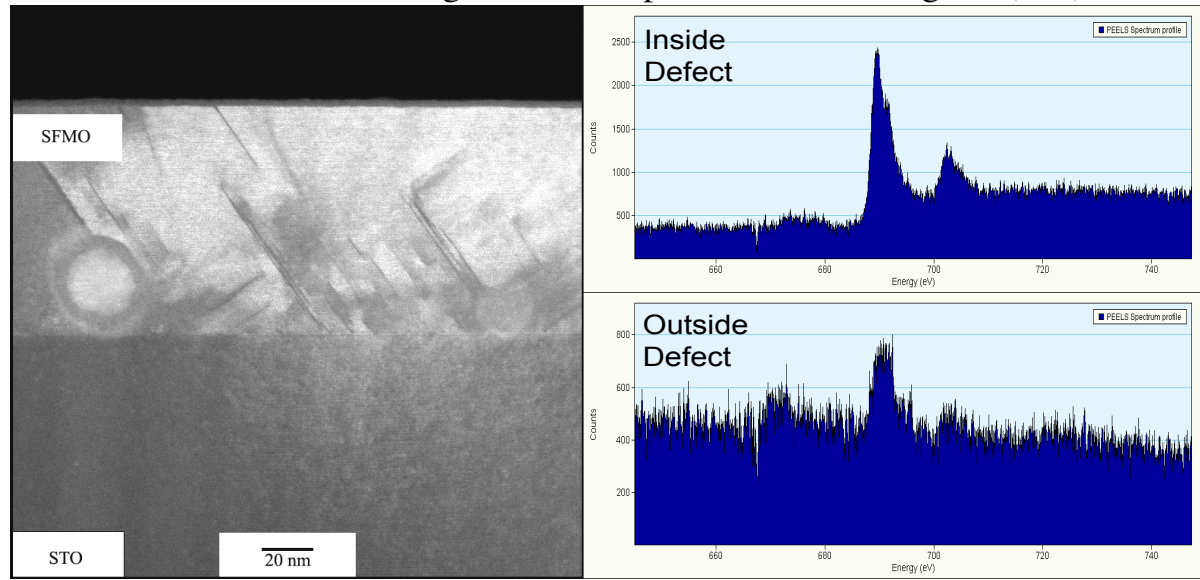

FIG. 3: HAADF-STEM image showing various defect structures as well as EELS data collected inside and outside of defects showing the variation in peak shape and bonding. 